\title{
REPRESENTATION OF HOLOMORPHIC FUNCTIONS BY BOUNDARY INTEGRALS
}

\author{
BY \\ ALBERT BAERNSTEIN II
}

\begin{abstract}
Let $K$ be a compact locally connected set in the plane and let $f$ be a function holomorphic in the extended complement of $K$ with $f(\infty)=0$. We prove that there exists a sequence of measures $\left\{\mu_{n}\right\}$ on $K$ satisfying $\lim _{n \rightarrow \infty}\left\|\mu_{n}\right\|^{1 / n}=0$ such that $f(z)=\sum_{n=0}^{\infty} \int_{K}(w-z)^{-n-1} d \mu_{n}(w)(z \in K)$. It follows from the proof that two topologies for the space of functions holomorphic on $K$ are the same. One of these is the inductive limit topology introduced by Köthe, and the other is defined by a family of seminorms which involve only the values of the functions and their derivatives on $K$. A key lemma is an open mapping theorem for certain locally convex spaces. The representation theorem and the identity of the two topologies is false when $K$ is a compact subset of the unit circle which is not locally connected.
\end{abstract}

1. Introduction. Let $K$ be a compact subset of the complex plane and let $\Omega$ be the complement of $K$ on the Riemann sphere, so that $\Omega$ is an open set which contains the point at infinity. Given complex Borel measures $\left\{\mu_{n}\right\}_{0}^{\infty}$ on $K$ which satisfy

$$
\lim _{n \rightarrow \infty}\left\|\mu_{n}\right\|^{1 / n}=0
$$

the function $f$ defined by

$$
f(z)=\sum_{n=0}^{\infty} \int_{K}(w-z)^{-n-1} d \mu_{n}(w) \quad(z \in \Omega)
$$

is holomorphic in $\Omega$ and vanishes at infinity.

In this paper we prove that, when $K$ is locally connected, the following converse holds:

THEOREM 1. Suppose that $K$ is a compact locally connected plane set. Then every function $f$ holomorphic in $\Omega$ satisfying $f(\infty)=0$ has a representation (2) with the measures $\left\{\mu_{n}\right\}$ satisfying (1).

Received by the editors April 20, 1970, and, in revised form, January 8, 1971.

AMS 1969 subject classifications. Primary 3035, 3085, 3030, 4630, 4690; Secondary 3021, 4620.

Key words and phrases. Representation of holomorphic functions, Cauchy integrals, boundary integrals, generalization of Laurent series, duality in function theory, spaces of holomorphic functions, Roumieu's generalized functions, open mapping theorem, $(D F)$ space, Montel space.

Copyright (c) 1971, American Mathematical Society 
When $K$ is a single point the representation asserted by Theorem 1 is just the Laurent expansion of $f$. For a general $K$, however, the measures $\mu_{n}$ will certainly not be uniquely determined by $f$.

The set $\Omega$ need not be connected. In fact it might have infinitely many components. "Holomorphic in $\Omega$ " means holomorphic in each component; there need be no analytic relation between the functions in different components.

If we start with an open set $G$ whose boundary $\partial G$ is locally connected and does not contain $\infty$, then each function $f$ holomorphic in $G$ has a representation (2) for $z \in G$ with $K=\partial G$. All we need do to prove this is extend $f$ in any way we like to a function holomorphic in the complement of $\partial G$, then use Theorem 1.

Johnson [4] proved a result similar to Theorem 1 for functions holomorphic in the unit disk. In $\$ 5$ we show that our representation is equivalent to his in this case, and answer (Theorem 3) a question of his concerning representability of functions holomorphic off a proper subset of the unit circle. Theorem 3(b) shows that Theorem 1 is false for arbitrary compact $K$.

In the proof of Theorem 1 a main idea is the duality between functions holomorphic on and off $K$ as developed by Köthe [5]. Consider the space $H(K)$ of (equivalence classes of) functions holomorphic in some neighborhood of $K$. The function $f$ of Theorem 1 induces a linear form on $H(K)$, which is continuous in the topology introduced by Köthe, denoted here by $\mathfrak{T}_{i}$. (Definitions are given in $\S 3$.) We will algebraically embed $H(K)$ in a space $X\left[\mathfrak{I}_{0}\right]$ whose topological dual consists of sequences of measures on $K$. Theorem 1 follows from the Hahn-Banach theorem once we show that the topology $\mathfrak{I}_{0}$ restricted to $H(K)$ is finer than the topology $\mathfrak{T}_{i}$. In $\S 2$ we prove an open mapping theorem which will imply that this is the case, and in $\$ 3$ we give the proof of Theorem 1. As a corollary of the proof we obtain in $\S 4$ a description (Theorem 2 ) of Köthe's topology $\mathfrak{I}_{i}$ which involves only the values of the functions and their derivatives on $K$.

We will be using the theory of locally convex spaces, and in particular inductive limits, whose basic properties we now recall. Let $E$ be a vector space and let $\left\{E_{n}\right\}$ be an increasing sequence of subspaces whose union is $E$. Suppose on each $E_{n}$ is a locally convex topology $\mathfrak{T}_{n}$ such that all the inclusions $E_{n} \rightarrow E_{n+1}$ are continuous. Let $\mathfrak{I}$ be the finest locally convex topology on $E$ for which all the inclusions $E_{n} \rightarrow E$ are continuous. Then $E[\mathfrak{T}]$ is called the inductive limit of the spaces $E_{n}\left[\mathfrak{T}_{n}\right]$. A 0 -neighborhood base in $E[\mathfrak{I}]$ is formed by the collection of all sets $\Gamma\left(\bigcup_{n=1}^{\infty} V_{n}\right)$ where $\Gamma$ means convex circled hull, and each $V_{n}$ is a zero-neighborhood in $E_{n}\left[\mathfrak{T}_{n}\right]$. A linear operator from $E[\mathfrak{T}]$ into a locally convex space is continuous if and only if its restriction to each $E_{n}\left[\mathfrak{T}_{n}\right]$ is continuous. For proofs see [2, p. 61]. (Read "circled" instead of "symmetric," and proofs are valid for complex case as well as real.)

2. A lemma from functional analysis. Consider the following situation. $E$ and $F$ are locally convex spaces and $T$ is a continuous linear mapping from $E$ into $F$. It is 
known that $T^{-1}(B)$ is bounded in $E$ whenever $B$ is a bounded subset of $F$. In particular, $T^{-1}(0)$ is bounded, hence $T$ is one-to-one. When can we assert that $T^{-1}$ is continuous?

We can do so if the range $T E$ is bornological, but this need not hold, even if $F$ is bornological and $T E$ is closed in $F$. Or, by Ptak's theorem [9, p. 163], we can do so if $E$ is $B_{r}$-complete and $T E$ is barreled. Again, however, it does not suffice that $E$ be $B_{r}$-complete and $F$ be barreled.

The following lemma gives conditions on $E$ and $F$ under which the answer is affirmative. A separated locally convex space is called a $(D F)$ space if it possesses a fundamental sequence of bounded sets and if each strongly bounded set in the dual which is the countable union of equicontinuous sets is itself equicontinuous.

LEMma. Suppose that $E$ is a separated locally convex space in which every closed bounded set is compact, and that $F$ is a $(D F)$ space. Let $T$ be a continuous linear mapping from $E$ into $F$ with the property that $T^{-1}(B)$ is bounded whenever $B$ is. Then $T^{-1}$ is a continuous linear mapping from $T E$ onto $E$.

Proof. We denote by $E^{\prime}$ and $F^{\prime}$ the topological dual spaces of $E$ and $F$, by $T^{\prime}$ the mapping adjoint to $T$, and by $A^{0}$ the polar of the set $A$ with respect to one of the dualities $\left\langle E, E^{\prime}\right\rangle$ or $\left\langle F, F^{\prime}\right\rangle$.

Let $B$ be a closed bounded convex circled subset of $F$. Then we have the equation $\left[T^{\prime}\left(B^{0}\right)\right]^{0}=T^{-1}(B)$. Take polars again. The left-hand side is the closure of $T^{\prime}\left(B^{0}\right)$ in the Mackey topology $\tau\left(E^{\prime}, E\right)$, and, since $T^{-1}(B)$ is assumed bounded, the right-hand side is a zero-neighborhood in the strong topology $\beta\left(E^{\prime}, E\right)$.

The hypothesis on $E$ implies in particular that every bounded set in $E$ is relatively weakly compact, so the topologies $\beta\left(E^{\prime}, E\right)$ and $\tau\left(E^{\prime}, E\right)$ coincide. Thus we have shown that the map $T^{\prime}: F_{\beta}^{\prime} \rightarrow E_{\beta}^{\prime}$ is nearly open. Since $F_{\beta}^{\prime}$ is a Fréchet space, in particular a Ptak space, it follows from [9, p. 163] that $T^{\prime}$ is a topological isomorphism. Thus $T^{\prime}\left(F^{\prime}\right)$, with the topology induced by $E_{\beta}^{\prime}$, is isomorphic to a quotient space of $F_{\beta}^{\prime}$, hence is a Ptak space, hence is complete. Since $T^{\prime}\left(B^{0}\right)$ is in fact dense in a zero-neighborhood for $E^{\prime}$ (not just for $T^{\prime}\left(F^{\prime}\right)$ ) it follows that $T^{\prime}\left(F^{\prime}\right)$ is dense in $E^{\prime}$, whence $T^{\prime}\left(F^{\prime}\right)=E^{\prime}$, and $E_{\beta}^{\prime}$ is thus a Fréchet space.

To prove that $T^{-1}$ is continuous, it suffices [9, p. 158] to show that each equicontinuous set $A$ in $E^{\prime}$ is the image by $T^{\prime}$ of an equicontinuous set in $F^{\prime}$. Since every bounded set in $E$ is relatively compact, hence precompact, it follows that every equicontinuous set in $E^{\prime}$ is precompact in $E_{\beta}^{\prime}\left[6\right.$, p. 268]. Since $E_{\beta}^{\prime}$ is complete, the strong closure of $A$ is compact, and thus [6, p. 281] of the form $T^{\prime}(C)$, where $C$ is a compact subset of $F_{\beta}^{\prime}$.

Since $C$ is precompact and $F_{\beta}^{\prime}$ is metrizable there is a null sequence whose (strongly) closed convex circled hull contains $C$ [6, p. 275]. Since $F$ is a $(D F)$ space, the null sequence is equicontinuous, hence so is its weakly closed convex circled hull. The weak closure contains the strong closure, thus $C$ is equicontinuous, and we are done. 
3. Proof of Theorem 1. For now, let $K$ be any compact set in the plane. Define the space $H(K)$ as follows: The function $g$ belongs to $H(K)$ if there exists an open set $U \supset K$ such that $g$ is holomorphic in $U$, and we identify two such functions if they agree on some neighborhood of $K$. For each $U$ as above, let $A(U)$ denote the space of functions holomorphic in $U$ and continuous on cl $U$, with topology defined by the norm $\|g\|_{U}=\sup _{z \in U}|g(z)|$. There is a natural map from $A(U)$ into $H(K)$. Let $\mathfrak{I}_{i}$ be the finest locally convex topology on $H(K)$ for which all these maps are continuous. Alternatively, $H(K)\left[\mathfrak{I}_{i}\right]$ can be described as the inductive limit of the spaces $A\left(U_{v}\right)$, where $\left\{U_{v}\right\}$ is any decreasing sequence of open sets containing $K$ such that each neighborhood of $K$ contains some $U_{v}$ and such that each component of each $U_{v}$ meets $U_{v+1}$.

The space $H(K)\left[\mathfrak{T}_{i}\right]$ is separated, a subset is bounded iff it is contained and bounded in some $A(U)$, and each closed bounded set is compact. Proofs may be found in [5] or in [6, p. 379].

Now, following Roumieu [7], let $X_{v}(\nu=1,2, \ldots)$ be the space of all sequences $h=\left\{h_{n}\right\}_{0}^{\infty}$, where each $h_{n}$ is a continuous function on $K$, and

$$
\|h\|_{\nu}=\sup _{n \geqq 0}\left\|h_{n}\right\| \nu^{-n}<\infty .
$$

(Here, and from now on, a norm without subscript means sup norm (or total variation, for measures) over $K$. Equipped with the norm \|\|$_{v}, X_{v}$ is a Banach space. Let $X=\bigcup_{v=1}^{\infty} X_{v}$. Let $\mathfrak{T}_{0}$ be the locally convex inductive limit topology induced on $X$ by the $X_{\nu}$.

The space $X\left[\mathfrak{I}_{0}\right]$ is separated. Each bounded set is contained and bounded in one of the $X_{v}$. For each continuous linear form $\varphi \in X^{\prime}$ there exists a sequence of measures $\left\{\mu_{n}\right\}_{0}^{\infty}$ on $K$ such that $\lim _{n \rightarrow \infty}\left\|\mu_{n}\right\|^{1 / n}=0$ and such that $\varphi(h)=\sum_{0}^{\infty} \int h_{n} d \mu_{n}$ $(h \in X)$.

The above statements are proved in [7, p. 43]. They can also be easily deduced from the proposition in our $\$ 4$. Moreover, $X$ is a $(D F)$ space, since it is the separated inductive limit of a sequence of normed, hence $(D F)$, spaces [3, Théorème 9].

Consider now the linear mapping $T: H(K) \rightarrow X$ defined by

$$
T g=\left\{(n !)^{-1} D^{n} g\right\}_{n=0}^{\infty} \quad(g \in H(K))
$$

where $D^{n} g$ is the restriction to $K$ of the $n$th (complex) derivative of $g$. If $U$ is an open set containing $K$ and $\nu$ is a positive integer with $\nu^{-1}$ less than the distance from $K$ to $\partial U$, it follows from Cauchy's formula that

$$
\|T g\|_{\nu} \leqq\|g\|_{U} \quad(g \in A(U)) .
$$

So $T$ restricted to each $A(U)$ is a continuous mapping into some $X_{v}$, hence into $X$, and it follows that $T$ is continuous on $H(K)\left[\mathfrak{I}_{i}\right]$.

We claim that

$$
T^{-1}(B) \text { is bounded in } H(K)\left[\mathfrak{I}_{i}\right] \text { whenever } B \text { is bounded in } X\left[\mathfrak{I}_{0}\right] \text {, }
$$
provided $K$ is locally connected. 
Let us assume that (1) is true. Then by the lemma of $\S 2, T^{-1}$ is continuous, and so $H(K)\left[\mathfrak{I}_{0}\right]$ coincides with $H(K)\left[\mathfrak{I}_{i}\right]$, where by $H(K)\left[\mathfrak{I}_{0}\right]$ we of course mean $H(K)$ regarded as a topological subspace of $X\left[\mathfrak{I}_{0}\right]$ (we will not distinguish $H(K)$ from $T(H(K)))$.

Now fix $f$ holomorphic in $\Omega$ with $f(\infty)=0$. For each open set $U$ containing $K$ select finitely many simple closed rectifiable curves in $U-K$ whose union $\gamma$ has the property

$$
\begin{aligned}
\int_{y}(z-w)^{-1} d z & =2 \pi i, & & w \in K, \\
& =0, & & w \notin U
\end{aligned}
$$

(for the existence of $\gamma$, see [8, p. 255]). For $g \in A(U)$ define

$$
\langle f, g\rangle=\frac{1}{2 \pi i} \int_{\gamma} f(w) g(w) d w .
$$

Then, for a given $U,\langle f, g\rangle$ does not depend on $\gamma$, and, for a given $g$, does not depend on the neighborhood $U$ in which $g$ is holomorphic. Letting $\varphi_{z}(w)=(w-z)^{-1}$ $(w \in K)$ where $z \in \Omega$ is fixed, we have $\left\langle f, \varphi_{z}\right\rangle=-f(z)$. The above assertions follow from Cauchy's theorem as stated in [1, p. 145].

When $U$ runs through all neighborhoods of $K$ the formula (2) defines a linear functional, denoted also by $f$, on $H(K)$. Since

$$
|\langle f, g\rangle| \leqq C\|g\|_{U} \quad(g \in A(U))
$$

where the constant $C$ depends only on $f$ and $U$, it follows that $f$ is continuous on $H(K)$ with the topology $\mathfrak{T}_{i}$, hence with the topology $\mathfrak{I}_{0}$. Extend $f$ by the HahnBanach theorem to an element of $X^{\prime}\left[\mathfrak{I}_{0}\right]$. Let $\left\{\mu_{n}\right\}$ be the measure sequence on $K$ associated with the extended functional. Then

$$
\langle f, g\rangle=\sum_{0}^{\infty} \int_{K} \frac{D^{n} g(w)}{n !} d \mu_{n}(w) \quad(g \in H(K)) .
$$

Theorem 1 follows from the choice $g(w)=\varphi_{z}(w)$ and an obvious change of notation.

Proof of (1). We can assume that $B$ is the unit ball in one of the $X_{v}$. Let $B_{v}$ denote its inverse image in $H(K)$ :

$$
B_{v}=\left\{g \in H(K):\left\|D^{n} g\right\| \leqq \nu^{n} n !, n=0,1,2, \ldots\right\} .
$$

We shall construct a neighborhood $V$ of $K$ into which all the elements of $B_{v}$ have (single valued) holomorphic extensions.

For each $w \in K$ choose $U_{w}$, open in $C$, such that $w \in U_{w} \subset \Delta\left(w, v^{-1}\right)$ and such that $K \cap U_{w}$ is connected. Here $\Delta(s, r)=\{z \in C:|z-s|<r\}$. Choose $r_{w}$ such that $\Delta\left(w, 2 r_{w}\right) \subset U_{w}$. Define $V=\bigcup\left\{\Delta\left(w, r_{w}\right): w \in K\right\}$. Take $g \in B_{v}$ and $z \in V$. Define

$$
g^{*}(z)=\sum_{n=0}^{\infty} \frac{D^{n} g(w)}{n !}(z-w)^{n}
$$


where $w$ is any point of $K$ such that $z \in \Delta\left(w, r_{w}\right)$. The series converges, since $|z-w|$ $<(2 v)^{-1}$. We have to show that $g^{*}(z)$ does not depend on $w$.

Suppose that $z \in \Delta\left(w, r_{w}\right) \cap \Delta\left(s, r_{s}\right)$, where $s, w \in K$. Assume that $r_{w} \leqq r_{s}$. Then $|s-w|<r_{w}+r_{s} \leqq 2 r_{s}$, hence $w \in \Delta\left(s, 2 r_{s}\right) \subset U_{s}$, hence both $s$ and $w$ belong to the connected set $U_{s} \cap K$. Let $U$ be an open set containing $K$ for which $g \in A(U)$. Then

$$
U_{s} \cap K \subset \Delta\left(s, \nu^{-1}\right) \cap U .
$$

Let $W$ be the component of the set on the right which contains $s$. Then $w \in W$.

The equation

$$
g(\tau)=\sum_{n=0}^{\infty} \frac{D^{n} g(s)}{n !}(\tau-s)^{n}
$$

is valid for all $\tau \in W$. Hence the series (3) around $w$ is a rearrangement of the series (4) around $s$, and uniqueness of $g^{*}(z)$ follows.

It is obvious that $g^{*}$ is holomorphic in $V$ and that $\left|g^{*}(z)\right|<2$ for all $z \in V$. It is easily verified that $g^{*}$ and $g$ agree on $V \cap U$, and hence define the same element of $H(K)$. Let $V_{1}$ be any open set with $K \subset V_{1} \subset \operatorname{cl~} V_{1} \subset V$. We have shown that

$$
B_{v} \subset\left\{g \in A\left(V_{1}\right):\|g\|_{V_{1}} \leqq 2\right\},
$$

which implies that $B_{v}$ is bounded in $H(K)\left[\mathfrak{T}_{i}\right]$.

4. The topology of $H(K)$. We use the notation of $\S 3$. The topology usually considered on $H(K), K$ compact, is the inductive limit topology $\mathfrak{I}_{i}$. For $K$ the unit circle, Johnson [4], using Fourier series, showed that this topology could be described without the intervention of the normed spaces $A(U)$. For $K$ any locally connected compact set we claim there is a description of $\mathfrak{I}_{i}$ in terms of certain "natural" seminorms. Let $\mathscr{A}$ be the class of all nonnegative sequences $\alpha=\left\{\alpha_{n}\right\}_{0}^{\infty}$ such that $\lim _{n \rightarrow \infty} \alpha_{n}^{1 / n}=0$. For $\alpha \in \mathscr{A}$ put

$$
p_{\alpha}(g)=\sum_{n=0}^{\infty} \alpha_{n}\left\|D^{n} g\right\|(n !)^{-1} \quad(g \in H(K))
$$

where \|\| denotes sup norm on $K$. Then we have the following theorem:

THEOREM 2. Let $K$ be a compact locally connected plane set. Then the topology $\mathfrak{I}_{i}$ on $H(K)$ coincides with the topology generated by the family of seminorms $p_{\alpha}$, $\alpha \in \mathscr{A}$.

Proof. From the proof of Theorem 1 we know that $\mathfrak{I}_{i}$ coincides with $\mathfrak{I}_{0}$, where $\mathfrak{I}_{0}$ is the topology $H(K)$ inherits when regarded as a subspace of $X$. So the proof of Theorem 2 reduces to proving the following analogous statement for $X$.

Proposition. The topology $\mathfrak{I}_{0}$ on $X$ coincides with the topology on $X$ generated by the family of seminorms $p_{\alpha}, \alpha \in \mathscr{A}$, where

$$
p_{\alpha}(h)=\sum_{n=0}^{\infty} \alpha_{n}\left\|h_{n}\right\| \quad(h \in X) .
$$


Proof. If $h \in X_{v}$ and $\alpha \in \mathscr{A}$ then $p_{\alpha}(h) \leqq\|h\|_{v} \sum \alpha_{n} \nu^{n}$. This proves that $p_{\alpha}$ is finite and that its restriction to each normed space $X_{v}$ is continuous. Since $p_{\alpha}$ is obviously homogeneous and subadditive, it follows that each $p_{\alpha}$ is a $\mathfrak{I}_{0}$-continuous seminorm.

Conversely, let $U$ be a $\mathfrak{I}_{0}$-neighborhood of 0 in $X$. We have to show that

$$
U \supset\left\{h \in X: p_{\alpha}(h) \leqq 1\right\}
$$

for some $\alpha \in \mathscr{A}$. We can assume that $U$ is the $\mathfrak{I}_{0}$-closure of $V$, where

$$
V=\Gamma \bigcup_{v=1}^{\infty}\left\{h \in X_{v}:\|h\|_{v} \leqq r_{v}\right\}
$$

( $\Gamma$ stands for convex circled hull) and the sequence $\left\{r_{v}\right\}$ is positive, but decreases so rapidly that $\lim _{v \rightarrow \infty} \nu^{n} r_{v}=0$ for each fixed positive integer $n$.

Define, for $n=0,1,2, \ldots$,

$$
\begin{aligned}
\beta_{n} & =\sup \left\{\nu^{n} r^{\nu}: \nu=1,2,3, \ldots\right\}, \\
\nu(n) & =\text { largest integer } \nu \text { for which } \beta_{n}=\nu^{n} r_{v} .
\end{aligned}
$$

Then for each $n$ we have $\beta_{n+1} \geqq[\nu(n)]^{n+1} r_{v(n)}$ hence

$$
\nu(n) \leqq \beta_{n+1} / \beta_{n} \text {. }
$$

The sequence $\nu(n)$ must tend to $\infty$ with $n$, because if not we would have $\nu(n) \leqq m$ for some integer $m$ and for all $n$ belonging to an infinite set $S$. This means that

$$
r_{v} v^{n} \leqq r_{v(n)}[\nu(n)]^{n} \leqq C m^{n} \quad(\nu \geqq m, n \in S)
$$

where $C$ is some constant larger than all the $r_{\nu}$. Fixing $\nu=m+1$ and letting $n \rightarrow \infty$, we get a contradiction.

It now follows from (1) that $\lim _{n \rightarrow \infty} \beta_{n}^{1 / n}=\infty$. Put $\alpha_{n}=2^{n} \beta_{n}^{-1}$. Then $\alpha=\left\{\alpha_{n}\right\} \in \mathscr{A}$. Suppose that $p_{\alpha}(h) \leqq 1$. Then $\left\|h_{n}\right\| \leqq 2^{-n} \beta_{n}(n=0,1,2, \ldots)$, hence

$$
\left\|h_{n}\right\| \leqq 2^{-n}[\nu(n)]^{n} r_{v(n)} \text {. }
$$

Let $h^{(n)}$ be the element of $X$ whose $n$th coordinate is $h_{n}$ and whose other coordinates are zero. Then $h^{(n)} \in X_{\nu}$ for all $\nu$ and $\left\|h^{(n)}\right\|_{\nu}=\nu^{-n}\left\|h_{n}\right\|(\nu=1,2,3, \ldots)$.

Taking $\nu=\nu(n)$ and using (2), we obtain

$$
\left\|h^{(n)}\right\|_{v(n)} \leqq 2^{-n} r_{v(n)} \quad(n=0,1,2, \ldots) .
$$

Thus

$$
\sum_{n=0}^{N} h^{(n)}=\sum_{n=0}^{N} 2^{-n}\left(2^{n} h^{(n)}\right)
$$

is an element of the set $V$ for each $N$.

We claim that

$$
\lim _{N \rightarrow \infty} \sum_{n=0}^{N} h^{(n)}=h
$$


where the limit is taken in the topology $\mathfrak{I}_{0}$. Let $j$ be an integer with $h \in X_{j}$. Then

$$
\left\|h-\sum_{n=0}^{N} h^{(n)}\right\|_{j+1}=\sup _{n \geqq N+1}\left\|h_{n}\right\|(j+1)^{-n} \leqq\|h\|_{j}(j / j+1)^{N+1}
$$

which tends to 0 as $N \rightarrow \infty$. Since injection from $X_{j+1}$ into $X$ is continuous, (3) follows. Hence $h$ belongs to the closure of $V$.

5. Functions holomorphic off a subset of the unit circle. Let

$$
H_{r}^{(n)}(\theta)=\frac{\partial^{n}}{\partial \theta^{n}} \frac{1+r e^{i \theta}}{1-r e^{i \theta}} \quad\left(n=0,1,2, \ldots, r \geqq 0, r e^{i \theta} \neq 1,0 \leqq \theta \leqq 2 \pi\right) .
$$

Johnson [4] proved that every function $f$ holomorphic in $|z|<1$ has a representation

$$
f(z)=\sum_{n=0}^{\infty} \int_{\Gamma} H_{r}^{(n)}(\theta-\varphi) d \lambda_{n}(\varphi) \quad\left(z=r e^{i \theta}, r<1\right)
$$

where the $\lambda_{n}$ are measures on the unit circle $\Gamma$ satisfying

$$
\lim _{n \rightarrow \infty}\left\|n ! \lambda_{n}\right\|^{1 / n}=0 .
$$

If all the $\lambda_{n}$ are supported in the closed subset $K \subset \Gamma$, then (1) defines a function holomorphic in $K^{c}$, the complement of $K$ on the Riemann sphere, which satisfies $f(0)=\lambda_{0}(\Gamma)=-f(\infty)$. Conversely, Johnson asks whether every function holomorphic in $K^{c}$ can be represented as a series (1) with all the measures supported in $K$, plus a constant.

The following result furnishes a complete answer to this question.

THEOREM 3. Let $K$ be a compact subset of $|z|=1$.

(a) Suppose that $K$ has only finitely many components. Then for each f holomorphic in $K^{c}$ there exists a sequence $\left\{\lambda_{n}\right\}_{0}^{\infty}$ of measures on $K$ satisfying (2), such that

$$
f(z)=\frac{1}{2}[f(0)+f(\infty)]+\sum_{n=0}^{\infty} \int_{K} H_{r}^{(n)}(\theta-\varphi) d \lambda_{n}(\varphi) \quad\left(z \in K^{c}\right) .
$$

(b) Suppose that $K$ has infinitely many components. Then the conclusion of (a) is false.

Thus the answer is affirmative if and only if $K$ is locally connected.

Proof of (a). Define polynomials $P_{n}(x)$, positive numbers $A_{m}^{(n)}$, and holomorphic functions $G^{(m)}$ by

$$
\begin{aligned}
P_{0}(x) & =1, & & \\
P_{n}(x) & =(n !)^{-1}(x+1)(x+2) \cdots(x+n) & & (n \geqq 1), \\
\sum_{m=0}^{n} A_{m}^{(n)} x^{m} & =P_{n}(x) & & (0 \leqq m \leqq n, n \geqq 0), \\
G(z) & =(1-z)^{-1}, \quad G^{(m)}=\partial^{m} G / \partial \theta^{m} & & \left(z=r e^{i \theta}\right) .
\end{aligned}
$$


Then, if $w=e^{i \varphi}$, we have

$$
\begin{aligned}
H_{r}^{(n)}(\theta-\varphi) & =2 G^{(n)}\left(z w^{-1}\right) & & (n \geqq 1) \\
& =2 G\left(z w^{-1}\right)-1 & & (n=0) .
\end{aligned}
$$

From

$$
\begin{aligned}
& G^{(m)}(z)=i^{m} \sum_{k=0}^{\infty} k^{m} z^{k} \quad(m \geqq 0), \\
& (1-z)^{-(n+1)}=\sum_{k=0}^{\infty} P_{n}(k) z^{k} \quad(n \geqq 0)
\end{aligned}
$$

for $|z|<1$ follows the identity

$$
(1-z)^{-(n+1)}=\sum_{m=0}^{\infty} A_{m}^{(n)} i^{-m} G^{(m)}(z) \quad(z \in C, z \neq 1) .
$$

We claim that

(6) If $\left\{\alpha_{n}\right\}_{0}^{\infty}$ is a nonnegative sequence satisfying $\lim _{n \rightarrow \infty} \alpha_{n}^{1 / n}=0$ and if $\beta_{m}$ $=\sum_{n=m}^{\infty} A_{m}^{(n)} \alpha_{n}(m \geqq 0)$ then each $\beta_{m}<\infty$ and $\lim _{m \rightarrow \infty}\left(m ! \beta_{m}\right)^{1 / m}=0$.

Proof of (6). For $n \geqq 0$ we have

$$
\begin{array}{ll}
P_{n}(x) \leqq \frac{2^{n}}{n !} x^{n} & (x \geqq n), \\
P_{n}(x) \leqq P_{n}(n) \leqq 4^{n} & (0 \leqq x \leqq n) .
\end{array}
$$

The first inequality follows at once from the definition (3); the second part of the second inequality is easily proved by induction.

Given $\varepsilon>0$ choose $B$ such that $\alpha_{n}<B \varepsilon^{n}$ for all $n$. Then for $x>0$ we have

$$
\begin{aligned}
\sum_{m=0}^{\infty} \beta_{m} x^{m} & =\sum_{m=0}^{\infty} x^{m} \sum_{n=m}^{\infty} A_{m}^{(n)} \alpha_{n}=\sum_{n=0}^{\infty} \alpha_{n} P_{n}(x) \\
& \leqq B \sum_{n \leqq x} \frac{2^{n} \varepsilon^{n} x^{n}}{n !}+\sum_{n>x} \alpha_{n} 4^{n} .
\end{aligned}
$$

The right-hand side is finite, hence so is each $\beta_{m}$. Moreover, as $x \rightarrow \infty$ the righthand side is less than $B \exp (2 \varepsilon x)+o(1)$, which implies that the power series with coefficients $\beta_{m}$ is entire and of exponential type zero, which implies $\left(m ! \beta_{m}\right)^{1 / m} \rightarrow 0$.

The set $K$ is locally connected. Apply Theorem 1 to the function $f(z)-f(\infty)$. Then

$$
f(z)=f(\infty)+\sum_{n=0}^{\infty} \int_{K}(w-z)^{-(n+1)} d \mu_{n}(w)
$$

with $\lim \left\|\mu_{n}\right\|^{1 / n}=0$. Define measures $\lambda_{m}$ on $K$ by

$$
d \lambda_{m}(w)=\sum_{n=m}^{\infty} A_{m}^{(n)} w^{-(n+1)} d \mu_{n}(w) \quad(m \geqq 0, w \in K) .
$$


Since $|w|=1$ on $K$ the measure $w^{-(n+1)} d \mu_{n}(w)$ has the same total variation as $d \mu_{n}$. So it follows from (6) that the series in (8) converges in norm, and, further, that the $\lambda_{m}$ satisfy the decay condition (2).

Fix $z \notin K$. Then, from (8),

(9) $\sum_{m=0}^{\infty} \int i^{-m} G^{(m)}\left(z w^{-1}\right) d \lambda_{m}(w)=\sum_{m=0}^{\infty} \sum_{n=m}^{\infty} i^{-m} A_{m}^{(n)} \int G^{(m)}\left(z w^{-1}\right) w^{-(n+1)} d \mu_{n}(w)$.

Each integral on the right has absolute value less than $\left\|\mu_{n}\right\| \sup _{w \in K}\left|G^{(m)}\left(z w^{-1}\right)\right|$ which is less than $m ! C^{m}\left\|\mu_{n}\right\|$, where the constant $C$ depends only on $z$ and $K$. (The inequality for $G^{(m)}$ is proved in [4].) It follows from (6) that

$$
\sum_{m=0}^{\infty} \sum_{n=m}^{\infty} A_{m}^{(n)} m ! C^{m}\left\|\mu_{n}\right\|<\infty,
$$

so the double series in (9) is absolutely convergent. Reversing the summation, then using (5) and (7), we obtain

$$
\sum_{m=0}^{\infty} \int i^{-m} G^{(m)}\left(z w^{-1}\right) d \lambda_{m}(w)=f(z)-f(\infty) .
$$

Replacing the $G^{(m)}$ by $H^{(m)}$ according to (4), making obvious notational changes, and using

$$
\begin{aligned}
H_{0}^{(n)}(\theta)=1 & \text { if } n=0, \\
& =0 \quad \text { if } n \geqq 1,
\end{aligned}
$$

we obtain the representation of $f$ asserted in (a).

Proof of (b). By a procedure like the one above, it can be shown that each function representable as in the conclusion of part (a) is also representable in the form (7). Therefore, it suffices to show that, whenever $K$ has infinitely many components, there exists a function $f$ holomorphic in $K^{c}$ which does not permit a representation (7) with $\left\|\mu_{n}\right\|^{1 / n} \rightarrow 0$.

Let $w_{0}=\exp \left(i \varphi_{0}\right)$ be a point of $K$ such that every neighborhood of $w_{0}$ meets infinitely many components of $K$. There is a positive sequence $\left\{\varphi_{m}\right\}_{m=1}^{\infty}$, decreasing to 0 , such that either

$$
\exp \left(i\left(\varphi_{0}+\varphi_{m}\right)\right) \in K(m \text { even }) \text { and } \exp \left(i\left(\varphi_{0}+\varphi_{m}\right)\right) \notin K(m \text { odd })
$$

or (10) holds with $+\varphi_{m}$ replaced by $-\varphi_{m}$. Because, if neither (10) nor the other statement were true, then $w_{0}$ would either be an isolated point of $K$, in the interior of an interval contained in $K$, or the endpoint of an interval of $K^{c}$. In each of these three cases every sufficiently small neighborhood of $w_{0}$ would have connected intersection with $K$, which violates the choice of $w_{0}$.

Say that (10) holds. From now on $m=2,4,6, \ldots$ Let $w_{m}=\exp \left(i\left(\varphi_{0}+\varphi_{m}\right)\right)$ and let

$$
K_{m}=\left\{\exp \left(i\left(\varphi+\varphi_{0}\right)\right) \in K: \varphi_{m+1}<\varphi<\varphi_{m-1}\right\}
$$


Choose, by Mittag-Leffler's theorem, a function $f$, holomorphic on the sphere except at the points $w_{m}$ ( $m$ even), which satisfies $f(\infty)=0$ and $\operatorname{Res}\left(f, w_{m}\right)=1$. Let $\Gamma_{m}$ be a small circle passing through $\exp \left(i\left(\varphi_{m+1}+\varphi_{0}\right)\right)$ and $\exp \left(i\left(\varphi_{m-1}+\varphi_{0}\right)\right)$. Then

$$
\begin{aligned}
\int_{\Gamma_{m}}(w-z)^{-1} d z=1 & & \text { if } w \in K_{m}, \\
& =0 & \text { if } w \in K-K_{m} .
\end{aligned}
$$

If $f$ possessed a representation of the form (7) we would have

$$
\int_{\Gamma_{m}} f(z) d z=\mu_{1}\left(K_{m}\right)
$$

so that $\mu_{1}\left(K_{m}\right)=2 \pi i(m=2,4,6, \ldots)$. Since $\mu_{1}$ is a finite measure and the $K_{m}$ are pairwise disjoint, the last statement is impossible.

\section{REFERENCES}

1. L. V. Ahlfors, Complex analysis: An introduction to the theory of analytic functions of one complex variable, 2nd ed., McGraw-Hill, New York, 1966. MR 32 \#5844.

2. N. Bourbaki, Espaces vectoriels topologiques, Actualités Sci. Indust., no. 1189, Hermann, Paris, 1953. MR 14, 880.

3. A. Grothendieck, Sur les espaces $(F)$ et $(D F)$, Summa Brasil. Math. 3 (1954), 57-123. MR 17, 765.

4. G. Johnson, Harmonic functions on the unit disc. I, Illinois J. Math. 12 (1968), 366-385. MR 37 \#5412.

5. G. Köthe, Dualität in der Funktionentheorie, J. Reine Angew. Math. 191 (1953), 30-49. MR 15, 132.

6. ——, Topologische Lineare Räume. I, Springer-Verlag, Berlin, 1960. MR 24 \#A411.

7. C. Roumieu, Sur quelques extensions de la notion de distribution, Ann. Sci. École Norm. Sup. (3), 77 (1960), 41-121. MR 22 \#12377.

8. W. Rudin, Real and complex analysis, McGraw-Hill, New York, 1966. MR 35 \#1420.

9. H. H. Schaefer, Topological vector spaces, Macmillan, New York, 1966. MR 33 \#1689.

Syracuse University,

SyRaCUSE, NeW YorK 13210 